\title{
THE R\&D PROGRAM FOR TARGETRY AT A NEUTRINO FACTORY
}

\author{
K.T. McDonald*, Princeton University, Princeton, NJ 08544, USA \\ K.A. Brown, C.C. Finfrock, D.M. Gassner, G.A. Greene, A.L. Hanson, S.A. Kahn, B.J. King, \\ H.G. Kirk, H. Ludewig, R.B. Palmer, A.F. Pendzick, R. Prigl, R. Samulyak, J. Scaduto, N. Simos, \\ P. Thieberger, T.Y.F. Tsang, H. Wang, R.J. Weggel, BNL, Upton, NY 11973, USA \\ Y. Fukui, UCLA, Los Angeles, CA 90095, USA \\ A. Bernadon, A. Fabich, C.D. Johnson, J. Lettry, H. Ravn, CERN, Geneva, Switzerland \\ N.V. Mokhov, FNAL, Batavia, IL 60510, USA \\ A. Zeller, Michigan State University, East Lansing, MI 48824, USA \\ D.L. Beshears, M.C. Cates, J.R. Haines, B.W. Riemer, C.-C. Tsai, ORNL, Oak Ridge, TN 37831, USA
}

\begin{abstract}
The need for intense muon beams for muon colliders [1] and for neutrino factories based on muon storage rings $[2,3,4]$ leads to a concept of 1-4 MW proton beams incident a moving target that is inside a 20-T solenoid magnet, with a mercury jet as a preferred example. Novel technical issues for such a system include disruption of the mercury jet by the proton beam and distortion of the jet on entering the solenoid, as well as more conventional issues of materials lifetime and handling of activated materials in an intense radiation environment. As part of the R\&D program [5] of the Neutrino Factory and Muon Collider Collaboration, R\&D effort related to targetry is being performed within the context of BNL E951 [6], first results of which are discussed here and in other contributions to this conference.
\end{abstract}

\section{THE TARGETRY CONCEPT}

A muon collider [1] or a neutrino factory based on a muon storage ring $[2,3,4]$ require intense beams of muons, which are obtained from the decay of pions produced in proton-nucleus collisions. To maximize the yield, pions of momentum near $300 \mathrm{MeV} / c$ should be captured [7, 8]. For proton energies above $10 \mathrm{GeV}$, the pion yield per unit of proton beam energy is larger for a high- $Z$ target [7]. For proton beam energies in the MW range, beam heating would melt or crack a stationary high- $Z$ target [9], so a moving target must be used. A mercury jet target is the main focus of BNL E951 [6], although R\&D is also being conducted on a carbon target option $[2,10,11]$ as might be suitable for a low-energy proton source [12], and conceptual studies have been carried out for rotating-band targets $[13,14]$, a tantalum/water target [15], and a liquid-lithium target [16].

The low-energy pions are produced with relatively large angles to the proton beam, and efficient capture into a decay and phase rotation channel is obtained by surrounding the target with a 20-T solenoid magnet, whose field tapers down to $1.25 \mathrm{~T}$ over several meters $[17,18]$, as sketched in

\footnotetext{
*mcdonald@puphep.princeton.edu
}

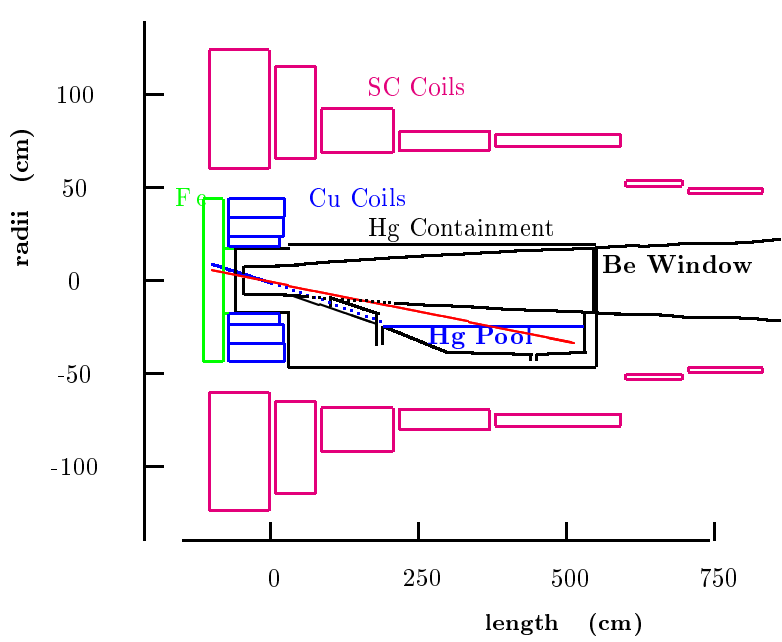

Figure 1: Concept of targetry based on a mercury jet and proton beam at $100 \mathrm{mrad}$ and $66 \mathrm{mrad}$, respectively, to the axis of a 20-T solenoid magnet.

Fig. 1. See also Figs. 2 and 3 of [8]. Pion yield is maximized with a mercury target in the form a $1-\mathrm{cm}$-diameter cylinder, tilted by about $100 \mathrm{mrad}$ with respect to the magnetic axis. To permit the proton beam to interact with the target over 2 interaction lengths, the proton beam is tilted by $33 \mathrm{mrad}$ with respect to the mercury jet axis.

The use of a mercury jet target raises several novel issues. The rapid energy deposition in the mercury target by the proton beam leads to intense pressure waves that can disperse the mercury [6, 19, 20, 21, 22, 23]. Further, as the mercury enters the strong magnetic field eddy currents are induced in the mercury, and the Lorentz force on these currents could lead to distortion of the jet $[6,23,24,25,26,27,28]$. On the other hand, the magnetic pressure on the mercury once inside the solenoid will damp mechanical perturbation of the jet [20, 29].

To address these issues an $\mathrm{R} \& \mathrm{D}$ program is now underway. 


\section{THE TARGETRY R\&D PROGRAM}

In the USA, R\&D on targetry for a neutrino factory and muon collider has been formalized as BNL experiment 951 [6]. This project maintains close contacts with related efforts in Europe [30] and in Japan [31].

The broad goal of E951 is to provide a facility that can test all the major of a liquid or solid target in intense proton pulses and in a 20-T magnetic field. A sketch of the eventual facility is shown in Fig. 2.

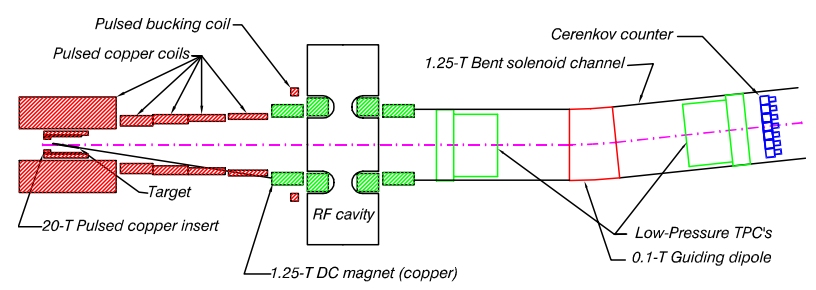

Figure 2: Sketch of the full configuration of BNL E951, the targetry R\&D facility.

Present activities in E951 focus on the interaction of intense proton pulses with targets in zero magnetic field. European targetry studies presently emphasize the interaction of mercury jets with a magnetic field, the operation of $\mathrm{rf}$ cavities near high-power targets [32], and evaluation of target materials [33].

\subsection{Mercury Target Studies}

The present $R \& D$ program on mercury jets is an outgrowth of work at CERN in the 1980's in which a prototype mercury jet was prepared (Fig. 3), but was never exposed to a beam.

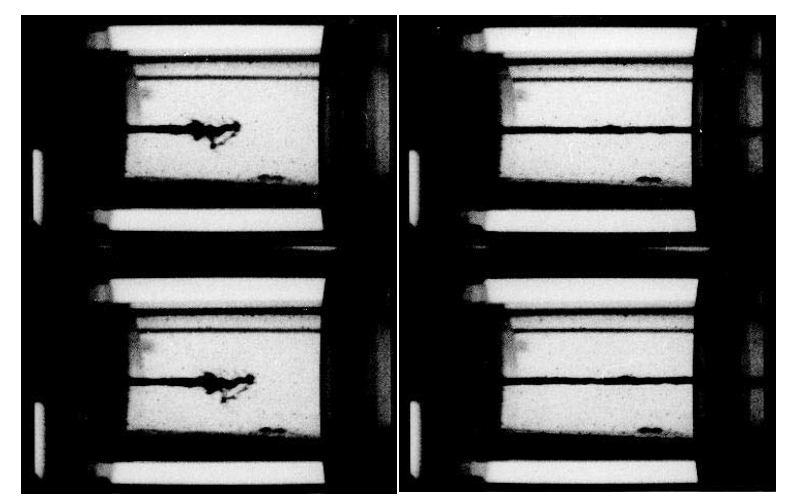

High-speed photographs of mercury jet target for CERN-PS-AA (laboratory tests) 4,000 frames per second, Jet speed: $20 \mathrm{~ms}-1$, diameter: $3 \mathrm{~mm}$, Reynold's Number: $>100,000$

Figure 3: Photographs of a 3-mm-diameter mercury jet (C.D. Johnson, 1988).

Experiment 951 is conducted in the A3 beamline of the BNL AGS [34] into which a single bunch of up to $5 \times 10^{12}$ $24-\mathrm{GeV}$ protons can be extracted and brought to a focus as small as $0.6 \times 1.6 \mathrm{~mm}^{2}$. The dispersal of both static and moving mercury targets by the proton beam was observed via two high-speed cameras using shadow photography with a laser diode [35]. The principal results obtained thus far in 2001 are summarized elsewhere [36, 37]. Figure 4 shows results from a static mercury target. Dispersal velocities of up to $50 \mathrm{~m} / \mathrm{s}$ were observed. The air in the target cell slowed the droplet velocity by a factor of two over $10 \mathrm{~cm}$. A key result from the jet studies was that the dispersal of mercury by the proton beam was confined to that part of the jet directly intercepted by protons.
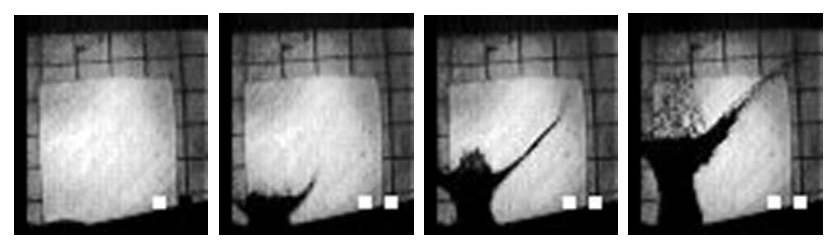

Figure 4: Exposures of $25 \mu \mathrm{s}$ at $t=0,0.5,1.6,3.4 \mathrm{msec}$ after a pulse of $2 \times 10^{12}$ protons interacted with a "thimble" of mercury $1.0 \mathrm{~cm}$ in diameter and $1.5 \mathrm{~cm}$ deep.

Thus, it appears that the dispersal of mercury by a proton beam is dramatic, but not violent, and that the dispersal will be a relatively modest issue for a target facility that operates at $15 \mathrm{~Hz}$ [38].

\subsection{Solid Target Studies}

E951 included exposures of several solid targets to the proton beam, using fiberoptic strain sensors with $500 \mathrm{MHz}$ bandwidth to characterize the transient response of the targets to the pressure waves induced by beam energy deposition [39]. As expected, a carbon-carbon composite target with thermal expansion coefficient of less that $10^{-6} / \mathrm{K}$ showed much less strain than an ATJ graphite target.

The issue of the rate of sublimation of carbon targets at the elevated temperatures $(>1900 \mathrm{C})$ caused by exposure to a 1-MW beam is under continuing laboratory study. Calculations indicate that a helium atmosphere can greatly extend the operation life of a carbon target against sublimation [40].

\section{REFERENCES}

[1] C.M. Ankenbrandt et al. (The Muon Collider Collaboration), Status of muon collider research and development and future plans, Phys. Rev. ST Accel. Beams 2, 081001 (1999), http://prst-ab.aps.org/abstract/PRSTAB/V2/i8/E081001/

[2] N. Holtkamp, D. Finley, eds., A Feasibility Study of a Neutrino Source Based on A Muon Storage Ring, http://www.fnal.gov/projects/muon_collider/nu-factory/

[3] S. Ozaki et al., eds., Feasibility Study-II of a Muon-Based Neutrino Source, http://www.cap.bnl.gov/mumu/studyii/FS2-report.html

[4] R. Palmer et al., A Second Feasibility Study of a Muon Storage Ring Neutrino Factory, PAC2001, FOAC007.

[5] M. Zisman, Status of Neutrino Factory and Muon Collider $R \& D$, PAC2001, WOAB008. 
[6] J. Alessi et al., An R\&D Program for Targetry at a Muon Collider, proposal to the BNL AGS (Sept. 1998); http://puhep1.princeton.edu/mumu/target/targetprop.ps http://puhep1.princeton.edu/mumu/target/pulsedjet.ps

[7] N. Mokhov, Particle Production and Radiation Environment at a Neutrino Factory Target Station, PAC2001, FOAC010; see also http://www-mucool.fnal.gov/mcnotes/muc0194.ps

[8] A. Hassanein et al., The Primary Target Facility for a Neutrino Factory Based on Muon Beams, PAC2001, TPAH155.

[9] R. Horne and C.D. Johnson, Post-Irradiation Examination of a High-Intensity Proton Beam Target, http://nicewww.cern.ch/ ${ }^{\sim}$ cdj/public/p-i-exam/pbartga4.htm

[10] B. Riemer, Graphite Rod Test/Simulation Response from LANSCE-WNR Proton Beam, http://puhep1.princeton.edu/mumu/target/graphitelansce.pdf

[11] B. Riemer, Stresses in ATJ Graphite Rod from AGS Beam, http://puhep1.princeton.edu/mumu/target/graphite_stress.pdf

[12] B. Autin et al., Design of a $2.2 \mathrm{GeV}$ Accumulator and Compressor for a Neutrino Factory, http://nicewww.cern.ch/ ${ }^{\sim}$ molat/neutrino/nf31.ps

[13] B. King et al., An Inconel Rotating Band Pion Production Target for Neutrino Factories and Muon Colliders, PAC2001, TPAH136; see also http://www-mucool.fnal.gov/mcnotes/muc0199.pdf

[14] J.R.J. Bennett, A High Power, Radiation Cooled Rotating Toroidal Target for Neutrino Production, http://puhep1.princeton.edu/mumu/target/bennett_011900.pdf

[15] P. Sievers, A Stationary Target for the CERN-Neutrino Factory, http://nicewww.cern.ch/ ${ }^{\sim}$ molat/neutrino/nf65.pdf

[16] V. Balbekov and N. Mokhov, Low-Budget Muon Source, PAC2001, TPAH144.

[17] R.J. Weggel, Conceptual Design of a Magnet System to Generate $20 \mathrm{~T}$ in a $0.15 \mathrm{~m}$ Diameter Bore, Employing an Inductor Precooled by Liquid Nitrogen, IEEE Trans. Appl. Superconductivity, vol. 11 ( Mar. 2000), http://puhep1.princeton.edu/mumu/target/mt16.ps

[18] R.J. Weggel et al., Design Study for $20 \mathrm{~T}, 15 \mathrm{~cm}$ Bore Hybrid Magnet with Radiation-Resistant Insert for Pion Capture, PAC2001, RPPH074.

[19] C.D. West, Cavitation in a Mercury Target, ORNL/TM2000/263, http://puhep1.princeton.edu/mumu/target/hg_cavitation.pdf

[20] P. Sievers and P. Pugnat, Response of Solid and Liquid Targets to High Power Proton Beams for Neutrino Factories, http://nicewww.cern.ch/ ${ }^{\sim}$ molat/neutrino/nf35.pdf

[21] N. Simos et al., Thermodynamic Interaction of the Primary Proton Beam with a Mercury Jet Target at a Neutrino Factory Source, PAC2001, RPAH076.

[22] A. Hassanein and I. Konkashbaev, Thermoelastic Response of Suddenly Heated Liquid and Solid Targets for High Power Colliders, PAC2001, RPAH071.

[23] R. Samulyak and W. Oh, Global Numerical Modeling of the Muon Collider Target, PAC2001, TPAH139.

[24] P. Thieberger, Estimated perturbations of the axial motion of a liquid-metal jet entering a strong magnetic field, http://www-mucool.fnal.gov/monotes/muc0182.pdf
[25] K.T. McDonald, Magnetohydrodynamics of a Continuous Mercury Jet Coaxially Entering a Solenoid, http://puhep1.princeton.edu//mumu/target/continuousjet.ps

[26] J. Gallardo et al., First order perturbative calculations for a conducting liquid jet in a solenoid, http://pubweb.bnl.gov/people/palmer/nu/study2/jet/jet.pdf

[27] S. Kahn et al., Calculations for a Mercury Jet Target in a Solenoid Magnet Capture System, PAC2001, ROPB010.

[28] A. Hassanein and I. Konkashbaev, Dynamics of Liquid Metal Jets Penetrating a Strong Magnetic Field, PAC2001, ROPB009.

[29] K.T. McDonald, Damping of Radial Pinch Effects, http://puhep1.princeton.edu/mumu/target/radialpinch.ps

[30] B. Autin, European $R \& D$, presented at NuFACT'01 (May 26, 2001),

http://www-prism.kek.jp/nufact01/May26/Plenary/26ple_autin.pdf

[31] K. Nakamura, An International Agreement for Neutrino Factory R\&D, presented at NuFACT'01 (May 26, 2001), http://www-prism.kek.jp/nufact01/May28/Plenary/28ple_nakamura.pdf

[32] N. Marseille and W. Pirkl, Sparking Cavity Test Report, http://nicewww.cern.ch/ ${ }^{\sim}$ molat/neutrino/nf52.pdf

[33] P. Drumm and C. Densham, Material Testing for Neutrino Factory Targets, PAC2001, TPAH157.

[34] K. Brown et al., First Beam Tests of the Muon Collider Target Test Beam Line at the AGS, PAC2001, TPAH129.

[35] T.Y.F. Tsang, E951 Diode Laser Operating Procedures, http://puhep1.princeton.edu/mumu/target/E951-laser-SOPv3.doc

[36] A. Fabich, Experimental Observation of MHD Effects and Proton Induced Shocks in Molten Metals, presented at NuFACT'01 (May 26, 2001), http://afabich.home.cern.ch/afabich/docs/NUFact01 talk.pdf

[37] H. Kirk et al., Target Studies with BNL E951 at the AGS, PAC2001, TPAH137.

[38] P. Thieberger, Mercury storm in the reaction chamber of the Muon Storage Ring and Collider project, http://www-mucool.fnal.gov/mcnotes/muc0212.pdf

[39] N. Simos et al., Thermal Shock Analysis of Windows Interacting with Energetic, Focused Beam of the BNL Muon Target Experiment, PAC2001, TPAH085.

[40] P. Thieberger, Upper limits for sublimation losses from hot Carbon targets in vacuum and in gases, http://www-mucool.fnal.gov/monotes/muc0186.pdf 